\title{
The Impact of COVID-19 Global Pandemic on Morbidity and Mortality of Liver Transplant Recipients Children and Adults: A Systematic Review of Case Series
}

\author{
Paschalis Gavriilidis, ${ }^{\mathrm{a}, \mathrm{b}}$, Madhava Pai ${ }^{\mathrm{a}}$
}

\begin{abstract}
The pandemic of coronavirus disease 2019 (COVID-19) changed the surgical everyday practice overnight. Currently, the first articles reporting outcomes of liver transplant recipients infected by severe acute respiratory syndrome coronavirus 2 (SARS-CoV-2) are published. The aim of the present study was to summarise the existing evidence of impact of COVID-19 global pandemic on liver transplant recipients. Electronic databases were searched in accordance with Preferred Reporting Items in Systematic Reviews and Meta-Analyses (PRISMA). Five studies were selected from a pool of 12 studies with a total of 854 liver transplant recipients of whom 700 were children and the rest 154 were adults. The present evidence, based on case reports and series demonstrated lower mortality in liver transplant recipients compared to general population.
\end{abstract}

Keywords: Coronavirus; COVID-19; Liver transplantation; Liver transplant recipients; Severe respiratory disease

\section{Introduction}

In December 2019, the coronavirus disease 2019 (COVID-19) was reported for the first time in Wuhan, Hubei province of China. The virus that causes the disease is named severe acute respiratory syndrome coronavirus 2 (SARS-CoV-2) [1]. On the March 12, 2020, the world health organization (WHO) declared COVID-19 as pandemic. Principal modes of spread reported were the droplet route and possible airborne spread. Of note, the virus was also transmitted during the incubation period by asymptomatic carriers $[2,3]$. Interestingly, the virus

Manuscript submitted May 18, 2020, accepted June 4, 2020

Published online June 25, 2020

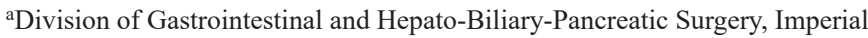
College Healthcare NHS Trust, Hammersmith Hospital, London, W12 0HS, UK

${ }^{\mathrm{b}}$ Corresponding Author: Paschalis Gavriilidis, Imperial College Healthcare NHS Trust, Hammersmith Hospital, London, W12 0HS, UK.

Email: pgavrielidis@yahoo.com

doi: https://doi.org/10.14740/jocmr4223 was also detected in the feces of patients whose respiratory samples were negative [4].

Common presenting symptoms are fever, nausea, anorexia, fatigue, nasal congestion, sore throat, myalgia, and diarrhea [1-3].

The number of reported cases of liver transplant patients with COVID-19 is very few and the reported clinical manifestation and symptoms do not differ from those of the general population. However, it is reported that organ transplant patients may manifest the disease with low-grade fever or no fever at all [5]. Therefore, increased awareness and vigilance are needed in the management of apyrexial transplanted patients.

So far there is no ample evidence to report any remarkable differences in the manifestation of disease in liver transplant patients compared to general population. The first fatal case of liver transplant patient due to SARS infection was reported in Toronto, Canada during the winter of 2003 [6]. In February 2020, the first fatal case in liver transplant recipient due to SARS-CoV-2 was reported in China. Consequently, case reports and series reported morbidity and mortality of liver transplant recipients tested positive for SARS-CoV-2 [7-11].

The aim of the present study was to summarise the reported evidence of liver transplant recipients infected by SARS$\mathrm{CoV}-2$ during the global pandemic. This systematic review was carried out in accordance with the guidelines set out in the Preferred Reporting Items in Systematic Reviews and MetaAnalyses (PRISMA) checklist [12].

\section{Literature Search}

A systematic literature search of articles published from inception until April 2020 performed in EMBASE, MEDLINE (PubMed), Cochrane Library, and Google Scholar databases using free text and MeSH terms (corona virus, COVID-19, liver transplantation, liver transplant recipients, global pandemic of COVID-19, severe acute respiratory syndrome, SARS). A grey literature search on www. clinicaltrials.gov was also performed. References cited in the retrieved articles were manually checked for further analysis. Disagreements between authors were resolved through discussion. 
Table 1. Study Characteristics

\begin{tabular}{|c|c|c|c|c|c|}
\hline Author/country & Number of LT patients & $\begin{array}{l}\text { Number of } \\
\text { tested positives }\end{array}$ & Age and gender & $\begin{array}{l}\text { Number of patients } \\
\text { with pneumonia }\end{array}$ & $\begin{array}{l}\text { Mortal- } \\
\text { ity }\end{array}$ \\
\hline $\begin{array}{l}\text { D'Antiga et al, } 2020 \\
\text { [7]/ Bergamo, Italy }\end{array}$ & 700 & 3 & Children & 0 & 0 \\
\hline \multirow{2}{*}{$\begin{array}{l}\text { Qin et al, } 2020[8] / \\
\text { Wuhan, China }\end{array}$} & 1 case report & 1 & 37 years old man & 1 & 0 \\
\hline & & & Perioperative infection & & \\
\hline $\begin{array}{l}\text { Bin et al, } 2020[9] / \\
\text { Wuhan, China }\end{array}$ & & & LT: July 17 & & \\
\hline $\begin{array}{l}\text { Huang et al, } 2020 \\
{[10] / \text { Fuzhou, China }}\end{array}$ & 1 case report & 1 & 59 years old man & 1 & 1 \\
\hline $\begin{array}{l}\text { Bhoori et al, } 2020 \\
{[11] / \text { Milano, Italy }}\end{array}$ & Short-term $<2$ years, 40 patients & S-T: 3 & S-T: NR & S-T: 0 & S-T: 0 \\
\hline \multirow[t]{3}{*}{ Total } & 854 patients & 12 & & 6 & $\begin{array}{l}4 \\
(2.60 \%)\end{array}$ \\
\hline & 700 children & & & & \\
\hline & 154 adults & & & & \\
\hline
\end{tabular}

LT: liver transplantation; L-T: long-term; S-T: short-term; NR: non-reported.

\section{Study, Selection, and Inclusion and Exclusion Criteria}

Studies that evaluated the impact of the COVID-19 pandemic on liver transplant recipients were included in this study. Abstracts and editorials without original data were excluded.

\section{Search Strategy and Included Study Character- istics}

Five studies were selected from a pool of 12 studies, included 854 liver transplant recipients of whom 700 were children and 154 adults [7-11] (Fig. 1, Table 1). Seven studies were excluded because of lack of original data.

According to Chinese data COVID-19 mortality is age dependent and stratified such as: age $50-59: 1.3 \%, 60-69$ : $3.6 \%, 70$ - $79: 8 \%, 80+: 14.8 \%$.

Three out of 700 children tested positive on SARS-CoV-2, and none developed pneumonia.

All deaths occurred in patients who transplanted more than 2.5 years ago and were in minimal immunosuppressive regimens.

\section{Discussion}

This is the first systematic review that estimated the impact of the COVID-19 global pandemic on morbidity and mortality of liver transplant recipients.

During the past two decades coronaviruses caused two global outbreaks those of SARS and Middle East respiratory syndrome (MERS). Recently, SARS-CoV-2 triggered the COVID-19 pandemic which is more infectious compared with previous ones due to asymptomatic transmission during the incubation period $[13,14]$.

The evidence regarding the impact of coronaviruses on liver transplant patients is scarce. In 2003, Kumar et al reported one fatal case in liver transplant recipient and consequently stratified the potential deceased donors in low, intermediate and high risk for SARS transmission based on clinical symptoms, hospital SARS exposure and epidemiological exposure [6]. It has been reported overall mortality of 3-4\% for SARS [15]. Multivariate analyses of the SARS outbreak from Hong Kong demonstrated that only advanced age was predictive of adverse outcome. Of note, they did not detect any specific comorbidity to be predictive of intensive care unit (ICU) admission or death [16].

Recent publications on liver transplant recipients based on case reports and series underline that conditions such as body mass index $>25$, history of cardiovascular event, arterial hypertension, hyperlipidemia and diabetes are predictive factors for adverse outcomes in patients tested positive with SARSCoV-2 $[3,7,11]$.

According to Chinese data COVID-19 mortality is age dependent and stratified such as: age 50 - 59: 1.3\%, 60 - 69: 


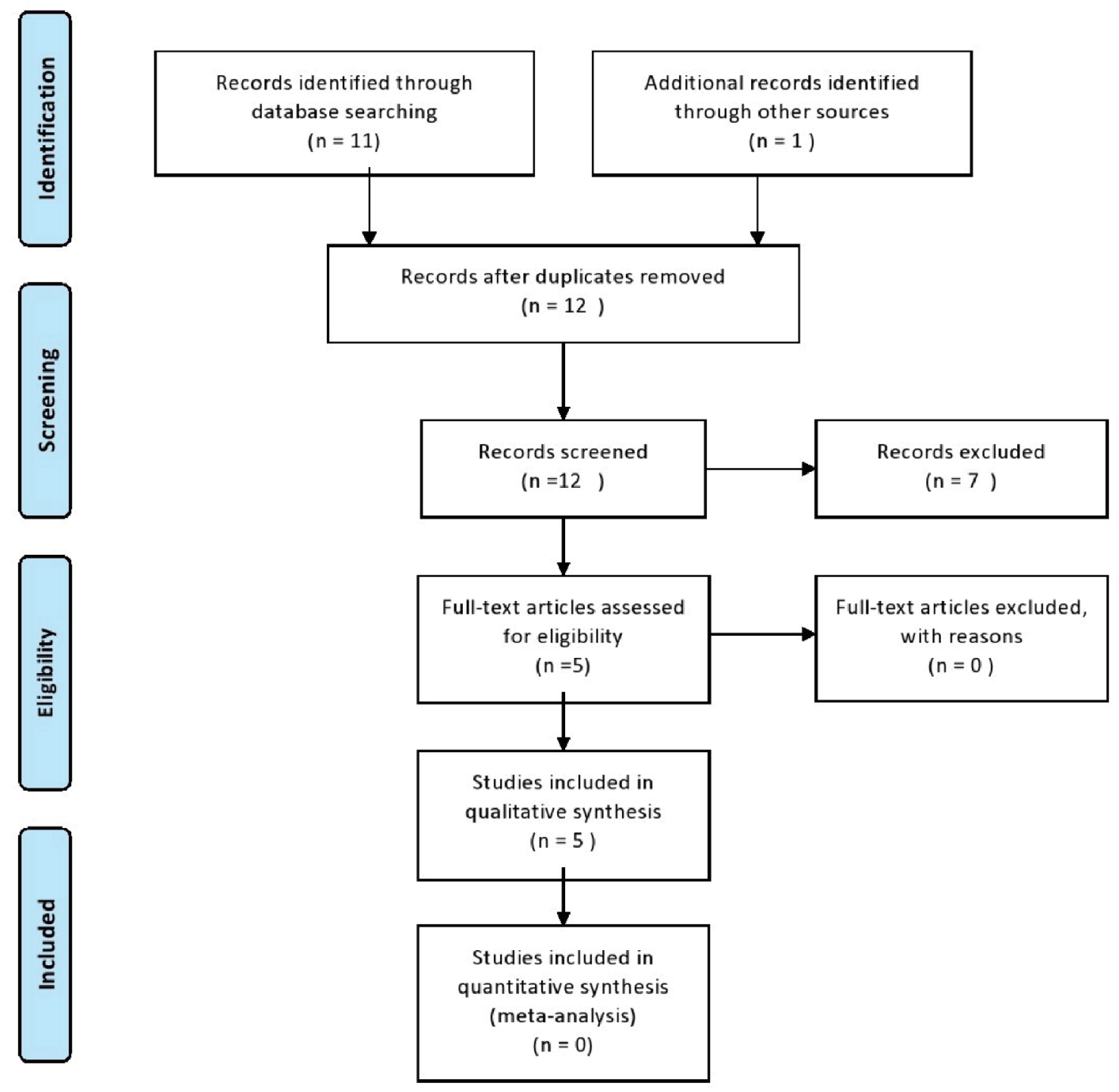

From: Moher D, Liberati A, Tetzlaff J, Altman DG, The PRISMA Group (2009). Preferred Reporting Items for Systematic Reviews and MetaAnalyses: The PRISMA Statement. PLoS Med 6(7): e1000097. doi:10.1371/journal.pmed1000097

For more information, visit www. prisma-statement.org.

Figure 1. Flow diagram of the search strategy.

$3.6 \%, 70-79: 8 \%, 80+: 14.8 \%$ [17]. In the present study, in the adult population of 154 patients four $(2.60 \%)$ deaths occurred; of those four cases, one patient was 59 years old and three over $65[10,11]$.

The immunosuppression levels to prevent rejection of liver transplants vary widely, not only between different patients, but also within the same patient over time.

Due to lack of criteria to determine the optimal level of immunosuppression required for each individual patient, it is objectively difficult to define immunosuppression minimization. The most common used definition is the following: "immunosuppression minimization is the state in which the immunosuppressive medications are decreased down to the levels that do not cause clinically significant side effects and yet prevent rejection" [18].

Another interesting topic that needs further investigation is whether immunosuppressed liver transplant patients are at increased risk to be infected more easily and to develop severe 
pneumonia compared to general population. Of note, D'Antiga reported that children liver transplant recipients although immunosuppressed were not at increased risk to develop severe COVID-19 compared with the general population [7]. The same author reported that three out of 700 children tested positive on SARS-CoV-2, and none developed pneumonia [7] (Table 1).

Qin et al [8] reported successful management of a fully immunosuppressed 37-year-old liver transplant recipient after perioperative infection with SARS-CoV-2. In addition, Bhoori et al reported that all deaths occurred in patients with minimal immunosuppressive regimens [11]. Further research needed to answer the question whether full immunosuppressive regimens mitigate the reactive innate immune response caused by SARS-CoV-2. In the present study, it is found that all deaths occurred in patients who transplanted more than 2.5 years ago and were in minimal immunosuppressive regimens $[10,11]$.

The results of the present study should be interpreted very cautiously because all the studies were case reports and series conducted in China and Italy with very short observational period. Therefore, national, institutional, underpowered sample, and detection bias might have influenced the results.

\section{Conclusions}

The present study demonstrated mortality rate in liver transplant recipients comparatively lower compared to general population. The fatal cases were patients over 65 years old and under minimal immunosuppressive regimens. Further clarification of the role and impact of minimal immunosuppressive regimens versus full immunosuppressive regimens of organ transplant recipients on the reactive innate immune response caused by SARS-CoV-2 would shed further light on the topic.

\section{Acknowledgments}

None to declare.

\section{Financial Disclosure}

None to declare.

\section{Conflict of Interest}

All named authors hereby declare that they have no conflict of interest to disclose.

\section{Author Contributions}

PG designed the research/study, performed research, collected data, analysed data, wrote the paper and did the critical review. MP designed research/study, performed research, analysed data, wrote the paper and did critical review.

\section{Data Availability}

The authors declare that data supporting the findings of this study are available within the article.

\section{References}

1. Zhu N, Zhang D, Wang W, Li X, Yang B, Song J, Zhao $\mathrm{X}$, et al. A novel coronavirus from patients with pneumonia in China, 2019. N Engl J Med. 2020;382(8):727733.

2. https://www.cdc.gov/coronavirus/2019-ncov/preventgetting-sick/how-covid-spreads.html.

3. Guan WJ, Ni ZY, Hu Y, Liang WH, Ou CQ, He JX, Liu L, et al. Clinical characteristics of coronavirus disease 2019 in China. N Engl J Med. 2020;382(18):1708-1720.

4. Xiao F, Tang M, Zheng X, Liu Y, Li X, Shan H. Evidence for gastrointestinal infection of SARS-CoV-2. Gastroenterology. 2020;158(6):1831-1833 e11833.

5. Liu H, He X, Wang Y, Zhou S, Zhang D, Zhu J, He Q, et al. Management of COVID-19 in patients after liver transplantation: Beijing working party for liver transplantation. Hepatol Int. 2020.

6. Kumar D, Tellier R, Draker R, Levy G, Humar A. Severe Acute Respiratory Syndrome (SARS) in a liver transplant recipient and guidelines for donor SARS screening. Am J Transplant. 2003;3(8):977-981.

7. D'Antiga L. Coronaviruses and Immunosuppressed Patients: The Facts During the Third Epidemic. Liver Transpl. 2020;26(6):832-834.

8. Qin J, Wang H, Qin X, Zhang P, Zhu L, Cai J, Yuan Y, et al. Perioperative presentation of COVID-19 disease in a liver transplant recipient. Hepatology. 2020.

9. Liu B, Wang Y, Zhao Y, Shi H, Zeng F, Chen Z. Successful treatment of severe COVID-19 pneumonia in a liver transplant recipient. Am J Transplant. 2020.

10. Huang JF, Zheng KI, George J, Gao HN, Wei RN, Yan $\mathrm{HD}$, Zheng $\mathrm{MH}$. Fatal outcome in a liver transplant recipient with COVID-19. Am J Transplant. 2020.

11. Bhoori S, Rossi RE, Citterio D, Mazzaferro V. COVID-19 in long-term liver transplant patients: preliminary experience from an Italian transplant centre in Lombardy. Lancet Gastroenterol Hepatol. 2020;5(6):532-533.

12. Moher D, Liberati A, Tetzlaff J, Altman DG, The PRISMA Group. Preferred reporting items for systematic reviews and meta-analyses: the PRISMA statement. PLoS Med. 2009;6(7):e1000097.

13. Peeri N, Shrestha N, Rahman M, et al. The SARS, MERS and novel coronavirus (COVID-19) epidemics, the newest and biggest global health threats: what lessons have we learned? Int J Epidemiol. 2020:1-10.

14. Yu P, Zhu J, Zhang Z, Han Y. A familial cluster of infection associated with the 2019 novel coronavirus indicating possible person-to-person transmission during the incubation period. J Infect Dis. 2020;221(11):1757-1761.

15. World Health Organization. Cumulative number of reported cases of Severe Acute Respiratory Syndrome 
(SARS). Available at: https://www. who.int.

16. Lee N, Hui D, Wu A, Chan P, Cameron P, Joynt GM, Ahuja A, et al. A major outbreak of severe acute respiratory syndrome in Hong Kong. N Engl J Med. 2003;348(20):19861994.

17. The Novel Coronavirus Pneumonia Emergency Response Epidemiology Team. The Epidemiological Character- istics of an Outbreak of 2019 Novel Coronavirus Diseases (COVID-19) - China, 2020. China CDC Weekly. 2020;2(8):113-122.

18. Londono MC, Rimola A, O'Grady J, Sanchez-Fueyo A. Immunosuppression minimization vs. complete drug withdrawal in liver transplantation. J Hepatol. 2013;59(4):872879. 